\title{
Immunonutrition: Modulating the immune response in critically ill and surgical patients through nutrition
}

\section{Scarlet Louis-Jean ${ }^{1}$ and Danik Martirosyan ${ }^{2}$}

${ }^{1}$ American University of Antigua College of Medicine, New York, NY, USA.; ${ }^{2}$ Functional Food Institute, Dallas, TX, USA

Corresponding author: Danik Martirosyan, PhD, Functional Food Institute, Dallas, TX, USA

Submission date: November $27^{\text {th }}$, 2018, Acceptance Date: November $29^{\text {th }}, 2018$, Publication Date: November $30^{\text {th }}, 2018$

Citation: Louis-Jean S and Martirosyan D., Immunonutrition: Modulating the immune response in critically Ill and surgical patients through nutrition. Bioactive Compounds in Health and Disease 2018; 1(7): 82-85. DOI: https://doi.org/10.31989/bchd.v1i7.587

\section{EDITORIAL}

Immunonutrition is a term given to nutritional interventions that modulate the immune and inflammatory responses in critically-ill and surgical patients. This is accomplished via the enteral or parenteral administration of formulas containing an array of immunonutrients in amounts greater than normally found in the diet. Some of the more commonly used immunonutrients include arginine, glutamine, branched-chain amino acids, omega-3 (n-3) fatty acids, trace metals (e.g. zinc, copper, iron), and nucleotides or antioxidants [1-3].

The human body's natural defense depends on the integrity of our immune system, which is responsible for curbing the course of pathogens, cancer cells, and their complications. The immune system is divided into two branches, innate and adaptive, which are responsible for responding to initial and recurrent infections respectively. Both branches are dependent on the use of mediators such as enzymes, pro-inflammatory cytokines, antibodies, and reactive oxygen and nitrogen species to fight many disease processes. The synthesis and functionality of these mediators are dependent on the individual components of immunonutrients [1].

The most often studied immunonutrients include arginine, glutamine, omega- 3 fatty acids, and nucleotides. The primary targets of these immunonutrients involve mucosal barrier function, cellular defense, and local or systemic inflammation [4]. Arginine is a conditionally essential amino acid whose synthesis is decreased during pathophysiological stress like sepsis and trauma. The immunomodulatory effects of arginine lie in its improvement of B and T-lymphocyte and macrophage functions. Arginine is also involved in many anabolic processes involved in growth and wound healing, as it participates in connective tissue synthesis, blood flow alteration, and angiogenesis. Glutamine is the most prevalent free amino acid in the body with various involvement in gluconeogenesis, renal ammoniagenesis, and intestinal mucosal integrity. Glutamine is also conditionally essential during catabolic conditions as it provides oxidative energy for dividing cells, increases antioxidant production, and acts as the primary fuel for cells of the intestines and the immune system $[3,5]$. 
Omega-3 (n-3) fatty acids exhibit anti-inflammatory effects by inhibiting the production of inflammatory eicosanoids (prostaglandins, leukotrienes, and thromboxanes). Arginine and n-3 fatty acids have been theorized to exhibit synergism, as the latter can decrease the expression of arginase I (arginine degrading enzyme), thereby increasing the availability of arginine. Nucleotides are necessary for de novo synthesis of DNA and RNA precursors, which are essential for energy transfer and synthesis of many proteins in the human body, especially in relation to cellular immune functions (e.g. cytokine \& antibody production and cellular proliferation) $[3,5]$.

Past research has demonstrated an intimate linkage between nutritional deficiency and impaired immune function. The correlation between malnutrition and infections are particularly salient in groups who are less immunocompetent, such as young children and the elderly, who are susceptible to higher rates of respiratory and digestive diseases. One study determined the enteral administration of glutamine decreased the incidences of moderate to severe sepsis and pneumonia in both premature infants and critically ill patients. Other studies claim supplementation of zinc at physiological levels for 1-2 months improves immune responses, decreases the incidence of infections, and ultimately increases survival [1].

In contrast, patients who have undergone significant physiological stresses such as surgery, trauma, or infection can experience deleterious effects from prolonged systemic inflammatory responses due to greater metabolic and essential nutrient demands. They may even experience compensatory immunosuppression as a result of chronic inflammation [4]. Many studies have shown varying combinations of immunonutrients can provide appropriate metabolic support to patients experiencing complications from illness-associated malnutrition, while effectively reducing the rates of infection and length of hospital stays. Additionally, other immunonutrients such as proteins, vitamins, trace metals, and enzymes exhibit antioxidant properties that limit the extent of tissue damage and reduce the likelihood of carcinogenesis [1,2].

In 2016, a non-blinded prospective, randomized study was conducted on the nutritional status and treatment toxicities of arginine, glutamine, and omega-3 fatty acids in head and neck cancer patients undergoing concurrent chemoradiotherapy (CCRT). The study recruited 40 patients, including 26 men and 14 women, matched by cancer type and baseline laboratory assessments. The patients were randomly assigned to two groups (A and B), each consisting of 20 participants. Despite an attrition of seven patients, an intention to treat analysis was conducted to include all collected data in the study [6].

Analysis of the results demonstrated severe grade 3-4 hematological toxicities were affected by 20 percent more compared to CCRT in patients who did not receive immunonutrition than patients who received immunonutrition. Additionally, patients receiving immunonutrition were more likely to complete their course of CCRT in a shorter period of time. In short, the study indicates immunonutrition combined with nutritional counseling is beneficial in reducing the severity in nutritional status deterioration and CCRT-associated toxicities in patients with head and neck cancers [6].

Nevertheless, skepticism surrounding the clinical effects of immunonutrition persist owing to wide heterogeneity and inconsistent results with regard to target populations, volumes of feeding delivered, and variation in formula composition [3, 4]. A systematic review conducted in 2018, reported no significant effect of immunonutrition on wound infection in 812 post-surgical head and neck cancer patients from 12 separate studies (risk ratio (RR) $0.94,95 \%$ CI 0.70 to 1.26) [7]. 
Additionally, studies have shown immunonutrition does not improve mortality and may even result in adverse outcomes in critically ill patients. Much of the issue surrounds the safety of arginine in septic patients. However, additional data is still needed as the results are not statistically significant [3]. Additional concern lies in whether the bioactive compounds of immunonutrition can be categorized as functional foods. Per the FFC, functional foods are defined as "natural or processed foods that contain biologically-active compounds; which, in defined, effective non-toxic amounts, provide clinically proven and documented health benefit utilizing specific biomarkers, for the prevention, management, or treatment of chronic disease or its symptoms" [8]. The FFC requires functional food products to meet the standards as outlined in the definition through the following steps [9]:

1. Examine the link between a particular food and health benefits.

2. Run in vitro and in vivo studies with non-living and animal specimens, respectively. Find the bioactive compound responsible for the health benefit.

3. Establish a daily value and possible mechanism of bioactive compound.

4. Run human studies. This involves administering human-appropriate dosages of Food Bioactive Compounds and testing for adverse side effects.

5. Develop appropriate food vehicles for bioactive compounds.

6. Obtain a special label for functional foods (with the information of daily usage amounts and length of usage).

7. Make approved health claims (FDA's Health Claims).

8. Market to the public and educate them about the health benefits of functional food.

9. Run studies on populations to test for long-term effects and overall product effectiveness. Measure public attitudes toward functional food.

Figure 1. FFC Steps for Meeting the Standards of Functional Food Products 


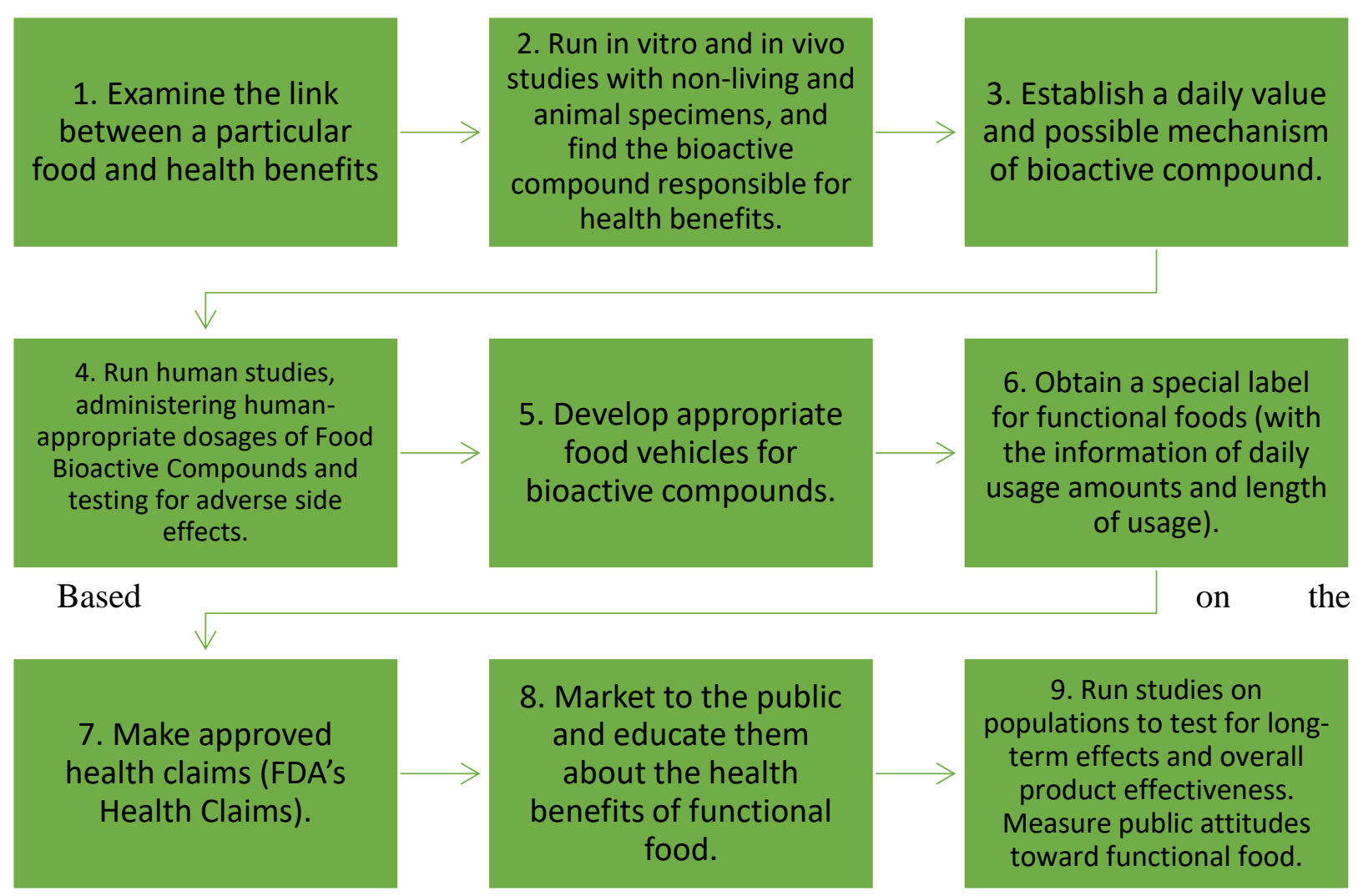

FFC's proposed standards for evaluating functional food and a 2016 review of published metaanalyses, future research on immunonutrition ought to be multidisciplinary and of a larger scale to better validate the vast benefits of immunonutrition, while providing data on optimal mixes and dosages for use in different patient groups $[4,9,10]$. More interventional studies are required to determine toxicities, confounding variables, and identify biomarkers, and ways by which immunonutrition can reduce mortality rates in critically-ill and surgical patients. Additionally, concerns of cost must be taken into consideration as the cost-effectiveness of immunonutrition therapies can vary based on specific populations, healthcare settings, and healthcare coverage [3].

\section{REFERENCES}

1. Vetvicka, V, Vetvickova, J: Concept of Immuno-Nutrition. Journal of Nutrition \& Food Sciences, 2016 06(03).

2. Grimble, R. F: Basics in clinical nutrition: Immunonutrition - Nutrients which influence immunity: Effect and mechanism of action. E-SPEN, the European E-Journal of Clinical Nutrition and Metabolism, 2008 4(1), 10-13. doi:10.1016/j.eclnm.2008.07.015

3. Roehl, K: Immunonutrition in 2016: Benefit, Harm or Neither? Practical Gastroenterology, 2016 154th ser., 27-40.

4. Calder, P. C: Immunonutrition may have beneficial effects in surgical patients. The BMJ,327(117), 2003 117-118.

5. Calder, P: Overview of nutrients with their functions and effects. The BMJ, 2003 327:117. 
6. Chang P, Yeh K, Wang C, Chen EY, Yang S, Huang J, et al.: Impact of the pretreatment Glasgow prognostic score on treatment tolerance, toxicities, and survival in patients with advanced head and neck cancer undergoing concurrent chemoradiotherapy. Functional Foods in Health and Disease, 2015 6(2), 121-132.

7. Howes N, Lewis SJ, Thomas S: Immunonutrition for patients undergoing surgery for head and neck cancer. Cochrane Database of Systematic Reviews, 2018 1-4.

8. Martirosyan D, Miller E: Bioactive Compounds: The Key to Functional Foods. Bioactive Compounds in Health and Disease 2018; 8(7):36-39

9. Zong J, Martirosyan DM: Anticancer Effects of Garlic and Garlic-derived Bioactive Compounds and its Potential Status as Functional Food. Bioactive Compounds in Health and Disease 2018; 1(2): 16-35

10. Osland E, Memon B, Memon MA: Pharmaconutrition administration on outcomes of elective oncological surgery for gastrointestinal malignancies: Is timing everything? a review of published meta-analyses until the end of 2016. Translational Gastroenterology and Hepatology, 2018 3(52): 1-8. 